\title{
A Web 2.0-based collaborative annotation system for enhancing knowledge sharing in collaborative learning environments
}

\author{
Addison Y.S. Su ${ }^{\text {a }}$, Stephen J.H. Yang ${ }^{\mathrm{a}, *}$, Wu-Yuin Hwang ${ }^{\mathrm{b}}$, Jia Zhang ${ }^{\mathrm{c}}$ \\ ${ }^{a}$ Department of Computer Science and Information Engineering, National Central University, No. 300, Jung-da Road, Chung-li, Tao-yuan 320, Taiwan, ROC \\ ${ }^{\mathrm{b}}$ Graduate School of Network Learning Technology, National Central University, No. 300, Jung-da Road, Chung-li, Tao-yuan 320, Taiwan, ROC \\ ${ }^{\mathrm{c}}$ Department of Computer Science, Northern Illinois University, DeKalb, IL 60115, USA
}

\section{A R T I C L E I N F O}

\section{Article history:}

Received 28 August 2009

Received in revised form

12 March 2010

Accepted 12 March 2010

\section{Keywords:}

Cooperative/collaborative learning Interactive learning environments

Authoring tools and methods

\begin{abstract}
A B S T R A C T
A limitation of current Web-based collaborative learning is the restricted ability of students to create and share individual annotations with annotated documents. Applying Web 2.0 collaborative annotation systems and analyzing students' annotation behavior has attracted attention to improve collaborative learning. This study designed a personalized annotation management system 2.0 (PAMS 2.0) for managing, sharing, and reusing individual and collaborative annotations as well as providing a shared mechanism for discussion about shared annotations among multiple users.

The purposes of this study are three-fold: (1) to understand students' perceived attitudes toward the use of PAMS 2.0; (2) to investigate the effects of different annotation sharing scenarios on quantity of annotation and its influence on learning achievements; and (3) to examine the relationship between learning achievements and quantity of annotation. A quasi-experiment was conducted with two classes of college students for fifteen weeks.

According to the results of the experiments, most of students in the experimental group are satisfied with the use of PAMS 2.0 for helping them create individual annotations and share their own annotations in collaborative learning context. Majorly students were interested in practical learning scenarios with PAMS 2.0 and thought this system is particularly useful for doing learning tasks. The analytical results of learning achievements show that the use of PAMS 2.0 can increase learning achievements in collaborative learning environments. Moreover, the results show that the influence of annotation on learning achievements becomes stronger with the use of the sharing mechanism.
\end{abstract}

(c) 2010 Elsevier Ltd. All rights reserved.

\section{Introduction}

The advancement of Computer Supported Cooperative Work (CSCW) has enabled a new style of collaborative learning called ComputerSupported Collaborative Learning (CSCL) (Koschmann, 1996; Ligorio \& Veermans, 2005; Wang, 2009; Zurita \& Nussbaum, 2004). The latest Web 2.0 technology has provided more opportunities to develop Web-based collaborative learning systems attracted learners to participate in collaborative learning context to learn from their collaborators (Barak, Herscoviz, Kaberman, \& Dori, 2009; Jones, Blackey, Fitzgibbon, \& Chew, 2010). Practically, collaborative learning is a social interaction that involving a community of learners and instructors, where members acquire and share experience or knowledge. Based on social constructivism, learners would learn more through the process of sharing experiences, discussion to build their knowledge (Vygotsky, 1978). From the social-cultural activity theory, the latest Web 2.0 technology can play a role as mediator for enriching technical courses with learning teams (Nardi, 1996). In fact, in Web 2.0-based systems, cross-platform environments and synchronous or/and asynchronous are all proper functions that provide learners with more equal opportunities for sharing information, retrieving information, and active interaction with other learners and instructors (Barak et al., 2009).

In traditional teaching and learning context, learners used to make marks on paper-based textbooks or handouts. Taking notes on textbooks or handouts is a common learning behavior. Furthermore, annotation offers a useful tool to increase deep reading of textual resource (Marshall, 1997, 1998), this is, the annotation causes a learner to think about the content that they are about to annotate in order to

\footnotetext{
* Corresponding author. Tel.: +8863422 7151x35326; fax: +8863422 2681.

E-mail addresses: addison@csie.ncu.edu.tw (A.Y.S. Su), jhyang@csie.ncu.edu.tw (S.J.H. Yang), wyhwang@cc.ncu.edu.tw (W.-Y. Hwang), jiazhang@cs.niu.edu (J. Zhang).
} 
ensure both the relevance and the worth of their thoughts before committing them to document. Robert (2009) mentioned that annotation made based on the profile of learners can be very useful in knowledge sharing and knowledge sharing in a collaborative environment can be drawn. Additionally, the unique features of annotation (e.g., catching attention, organizing, indexing, and discussing) have started increase attention in different research fields (Barak et al., 2009; Jones et al., 2010; Marshall \& Brush, 2004; Robert, 2009). Learners catch the attention of group members using annotations to help them focus on specific document content. Learners can also organize the document content with annotations, and use indexes or reminders for later reference. By reviewing others' thoughts in the form of annotations, learners can collaboratively explore and exploit valuable knowledge. Experienced instructors or learners may also provide valuable input to facilitate learners understand the material.

In order to provide these supports, the present study investigates how a Personalized Annotation Management System 2.0 (PAMS 2.0), as a Web 2.0 collaborative annotation system, helped students annotate documents and share their own annotations in collaborative learning context. Furthermore, PAMS 2.0 can help students coordinate and negotiate with others in face-to-face interaction and help instructors to understand student learning behaviors. A quasi-experiment was conducted with two classes of college students for fifteen weeks. According to the results of the experiments, most of students in the experimental group are satisfied with the use of PAMS 2.0 for helping them create individual annotations and share their own annotations in collaborative learning context. Majorly students were interested in practical learning scenarios with PAMS 2.0 and thought this system is particularly useful for doing learning tasks. The analytical results of learning achievements show that the use of PAMS 2.0 can increase learning achievements in collaborative learning environments. Moreover, the results show that the influence of annotation on learning achievements becomes stronger with the use of the sharing mechanism.

The remainder of this paper is organized as follows. Section 2 describes a brief survey of related studies. Section 3 presents how we built a PAMS 2.0 and how PAMS it can be used to facilitate collaborative annotation management, respectively. Section 4 describes the methodology of this research to evaluate whether or not different learning scenarios actually facilitates learning achievements in collaborative learning context. Section 5 presents experimental results and discusses findings. Finally, Section 6 draws conclusions and indicates directions for future research.

\section{Literature review}

In this section, the literature of theories of collaborative and Web-based collaborative learning as well as its importance in terms of annotation and annotation systems is discussed. Furthermore, the TAM (Technology Acceptance Model) model, which is adopted in this research to evaluate how users come to accept and use PAMS 2.0, is also introduced.

\subsection{Theories of collaborative and Web-based collaborative learning}

The success of collaborative learning activities requires the constant generation, transfer, and understanding of knowledge, making collaboration an essential and highly valued process. Furthermore, collaborative learning might enrich students' learning experiences by motivating them to seek new insights and perspectives. Studies track and analyze interaction patterns to guide collaborative learning. Collaborative learning is based on the constructivist theory of learning, which asserts that people are active learners and must construct knowledge for themselves (Geary, 1995) via interaction between individuals and an environment (Schunk, 1996). Avouris, Komis, Margaritis, and Fiotakis (2004) design an environment for monitoring and examining group learning patterns from two aspects: activity analysis and collaboration analysis. Collaboration feedbacks are also used to enhance collaborative learning (Zumbach, Hillers, \& Reimann, 2003). Bravo, Redondo, Ortega, and Verdejo (2006) build a learner-centered synchronous CSCL environment for students to study design and report a case study.

Liaw, Chen, and Huang (2008) reported that Web-based collaborative learning systems allow more opportunities for learners to participate without the limitation on knowledge levels. In Web-based collaborative learning context, Kagan (1994) pointed out students' ideas about collaborative learning and their experience with the use of technology might inhibit or promote their participation in the collaborative learning process. Furthermore, a collaborative task requires collaborators not only to share information, but also to discuss or determine how to rank other collaborator work. Dewiyanti, Brand-Gruwel, Jochems, and Broers (2007) also mentioned that learners are encouraged to exchange ideas, share perspectives, and use previous knowledge or experience in order to decide on the best solution for the question. Dori and Herscovitz (1999) suggested that the central role of education should be to develop in learners an appreciation of posting questions. In the research reported here, students were asked to develop questions relating to the course learning contents. By the use of Web-based collaborative learning systems, students were asked to share these questions with their collaborators, accumulate those questions as a form of preparing for the post-test, and evaluate questions posted by their collaborators.

In short, collaborative learning strategies (e.g., group learning, group investigation, jigsaw cooperative learning strategy, and dyadic cooperative learning strategy) can be applied to many instructional scenarios (Bravo et al., 2006; Hwang, Wang, \& Sharples, 2007; Wang, 2009). Based on the collaborative learning strategies, our experiment was organized by collaborative learning activities and instructional tools were provided to support the arranged different learning scenarios.

\subsection{Annotation and annotation systems}

Several e-learning models have proposed to describe learning content and annotations, such as Dublin Core (Dublin Core, 2001), LOM (LOM, 2002), and SCORM (SCORM, 2003). To avoid duplication and reinvention wherever possible, the proposed annotation model is derived from the Dublin Core. In contrast to previous studies (Yang, Chen, \& Shao, 2004), which model various properties of annotation metadata serving different purposes (either for a more generic purpose or for a specific purpose such as e-learning), this study focuses on how to store, manage, and retrieve annotation metadata by exploiting the latest Web 2.0 technology. Barak et al. (2009) thought the need for any proposed e-learning models to allow instructors or learners to specify the access level of individual annotations. This would increase the usefulness of the annotation system by removing those annotations where there is very little public relevance from the common pool, while retaining the ability to create such annotations (Marshall \& Brush, 2002). 
Much annotation systems have been proposed during the last decade. The Word Wide Web Consortium has proposed a Web-based annotation system called Annotea (2001). Annotea improves collaboration by sharing annotations, bookmarks, and their combinations. Microsoft has published OneNote that is a personalizable annotation tool. Users can use a digital pen, a keyboard, or a voice recording to input annotations. However, annotations can only be shared through e-mails. LeeTiernan and Grudin (2001) proposed a prototype that allows viewers of audio and video to create and share textual annotations that are synchronized with the multimedia. Researchers found that group practices can increase engagement and system use, and also suggest possible further improvement to the annotation system. Nokelainen, Kurhila, Miettinen, Floreen, and Tirri (2003) proposed a document-based annotation system for shared their own annotations. The usefulness of the annotation system was evaluated in a real-life collaborative learning context. Yang et al. (2004) proposed a personalized annotation management system. Researchers also demonstrated that personalized annotation enhances knowledge sharing and learning in students' online learning activity. The overall results indicated that learners who are willing to do real task with the tools provided by the annotation system, and are able to elaborate what they are doing, produce both highest quality annotations and learning achievements.

Recent studies pointed out the usefulness of annotation systems on facilitating learning. Nokelainen et al. (2003) indicated that annotation systems can bring about students' learning motivation, and they want to use the systems elaborated more about their tasks. Hwang et al. (2007) designed several sharing activities in which annotation increase students' learning efficiency. Researchers believe that it is not easy to underhand annotation systems for learning without considering their interaction with annotation sharing strategies. Marshall and Brush (2004) also suggested that knowledge sharing strategies provide an opportunity to facilitate making individual knowledge to public ones. Thus, knowledge accumulated its value when it is shared.

From previous studies (Yang et al., 2004), by analyzing the annotations made to a shared document, it would be possible to infer classification information related to the document. This classification information could assist in making the resource machine-understandable and a part of the Semantic Web technology to provide an increase in the significance of information presented as search results. Additionally, Azouaou, Chen, and Desmoulins (2004) proposed three principles to use in developing annotation systems for e-learning: usefulness, shareability, and usability. In online learning context, annotation systems should take the annotation sharing strategies into consideration. For example, annotation systems can cooperate with the learning activities and correspond to learning objectives. Secondly, annotation systems can foster learning with collaborative annotations through the annotation sharing strategies. Thirdly, annotation systems require a user-friendly interface, offering participants an easy means to interact with others while not hindering the interaction between online materials and annotations. The three principles directly motivate the present study, which upgrades PAMS 1.0 and integrates knowledge sharing strategies with the latest Web 2.0 technology to form our PAMS 2.0.

\subsection{Technology acceptance model: TAM}

The TAM (Technology Acceptance Model) was developed by Davis (1989) to evaluate how users come to accept and use a technology. Based on user acceptance of the technology, TAM theory proposes perceived usefulness and perceived ease of use to explain a user's attitude toward a system. As a result of TAM related research, perceived usefulness was further employed to study a user's intention to use the system, and it was found that perceived ease of use also had a significant influence on the perceived usefulness of a user who is currently using or learning an information technology. This study employs TAM theory to explore the "perceived usefulness" and "perceived ease of use" of PAMS 2.0.

\section{Design and development of the PAMS 2.0 system}

\subsection{The system architecture}

PAMS 2.0 adopts a client/server architecture, where the system architecture comprises two separate parts: an annotator side and a server side.

On the server side, four managers provide four system-level supporting services: an anchoring position mechanism, a document manager, an association manager, and a user manager. Four repositories store detailed information regarding annotations, annotated objects, annotated documents, their associations, and corresponding users. These repositories are the annotation repository, the annotated document repository, the association repository, and the user repository.

On the annotator side, four handlers collaborate to process annotation creation, retrieval, discussion, and management. Annotators can create, edit, and retrieve their own annotations. Additionally, users can retrieve the annotations from other users in the same group. Moreover, users can discuss with each other through the annotation discussion handler. Access control is handled by the user, using a predefined set of roles. In the current version of PAMS, we realize a straightforward access control. An annotated document can only be freely annotated by the members of a predefined group. The creator of a specific annotation may constrain that only certain roles in the annotator group have access to the annotation. New annotators may join in the group in the rum-time. More comprehensive and customizable access control mechanism will be our future research topic.

\subsection{Annotation modeling}

\subsubsection{Representation of annotation management model}

This system developed a novel annotation management model that specifies the six entity types (user, group, role, annotation, annotated object, and annotated document) and two relationships (association and assignment) as shown in Fig. 1.

The annotation management model is defined as follows. A document with annotations is called an annotated document. Annotations can be created by any user (author) on any part (area) in the document, such as a word, a sentence, a section, or even the entire document. Any part of the document associated with an annotation is called an annotated object. Annotated documents can allow a role-based access control (Sandhu, Coyne, Feinstein, \& Youman, 1996) (e.g., a learner may have read and write privileges to annotations and annotated 


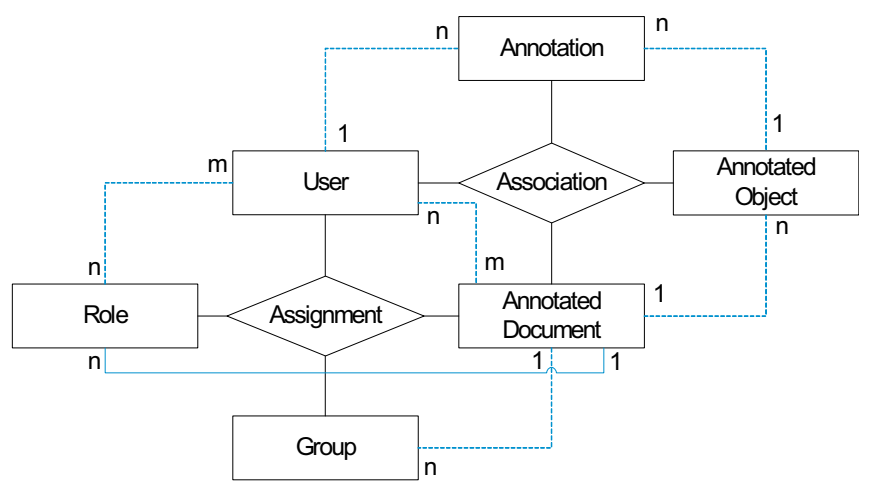

Fig. 1. Annotation management model.

documents). All users (authors) with the same access privilege (role) to a particular annotated document can form groups. The relationship between a specific annotation with its corresponding annotated object and annotated document is recorded in an association. The relationship between a specific user and its corresponding annotated document, user, group, and role is recorded in an assignment. Furthermore, the relationship properties are able to help users in managing, reusing, and sharing their own annotations.

\subsubsection{Storage of annotation management model}

This annotation management model has three metadata (annotation metadata, annotated object metadata, and annotated document metadata) derived from Dublin Core for annotation management.

The annotation metadata includes eight basic attributes that describe its primitive properties. Each annotation is assigned a unique identifier in the domain of an annotated document. Typically, an annotation requires a title and a description. Its title can be used for indexing and searching, while its description is only retrieved upon request. It comprises four types of annotation: (1) definitions (e.g., descriptions and explanations), (2) comments (e.g., opinions and arguments), (3) questions (e.g., problems and answers), and (4) associations (e.g., links to other resources). A user can provide definitions on an annotated object in an annotated document, raise questions, or make specific associations between annotated objects. Without losing generality, an annotation can be represented in text, graphics, or voice formats. Additionally, a user may handwrite annotations; the original version and its transformed electronic format are both stored. Each annotation stores its user's name to retrieve users' annotations. Finally, each annotation is associated with an annotated object in the corresponding annotated document. The anchoring position of the annotation object is also annotated.

The annotated object metadata includes five basic attributes describing the primitive properties of an annotated object encompassed in an annotated document. Each annotated object is assigned a unique identifier in the domain of an annotated document. Without losing generality, the annotated object format includes three kinds of annotated objects: text, image, or audio. An annotated object can be associated with a short description. Each annotated object must carry its anchoring position in the corresponding document and document identifier.

The annotated document metadata includes ten basic attributes describing the primitive properties of an annotated document. Each annotated document is assigned a unique identifier. An annotated document typically carries a set of properties, including title, short description, creator, publisher, publication date, URI on the Internet, type, and format, and language used in the document.

\subsection{Annotation creation}

Fig. 1 shows that annotations are maintained separately in the association property, without affecting the original document. While this decoupled approach provides flexible viewing (through different association properties) and higher security (as the original annotated documents remain intact), a major challenge is how to dynamically associate relevant annotations to the corresponding annotated objects in specific annotated documents. The concept of anchoring position can be used to solve this problem.

This system builds an anchoring position mechanism, which implements annotation integration using lazy initialization. This mechanism is stored in an eXtensible Stylesheet Language (XSL) file, which supports styling functions. With the AJAX technique, annotations can be dynamically retrieved and incorporated into the corresponding annotated documents during browsing without modifying the original copy. The following stepwise process illustrates how to make an anchoring position while creating an annotation.

Step 1 Retrieve the metadata of the annotated object in a special document (document_URI).

Step 2 Specify the desired annotated object (object_id).

Step 3 Compute the anchoring position as a tuple comprising a starting and an ending position of the anchor (anchor_position) based on the specified object (object_id).

Step 4 Handwrite an annotation (annotation_INK) by using the annotation creation handler.

In Fig. 2, the anchoring position represents a spatial association that positions an annotation in a specific document. When an annotation $A N$ is annotated on a corresponding object $A O$ in a document $A D$, where $A N \in A, A O \in O$, an association is generated as a 3-tuple (AN\#, $A U$, $A O$ ) relationship, and $\mathrm{AN \#}$ is the serial number of annotation $\mathrm{AN}, \mathrm{AU} \in \mathrm{U}$. For example, if a document is a typical academic article, which contains several special objects such as paper title, author, abstract, keyword, and section title. The annotation association AN(\#1,Vincent,AO1) 


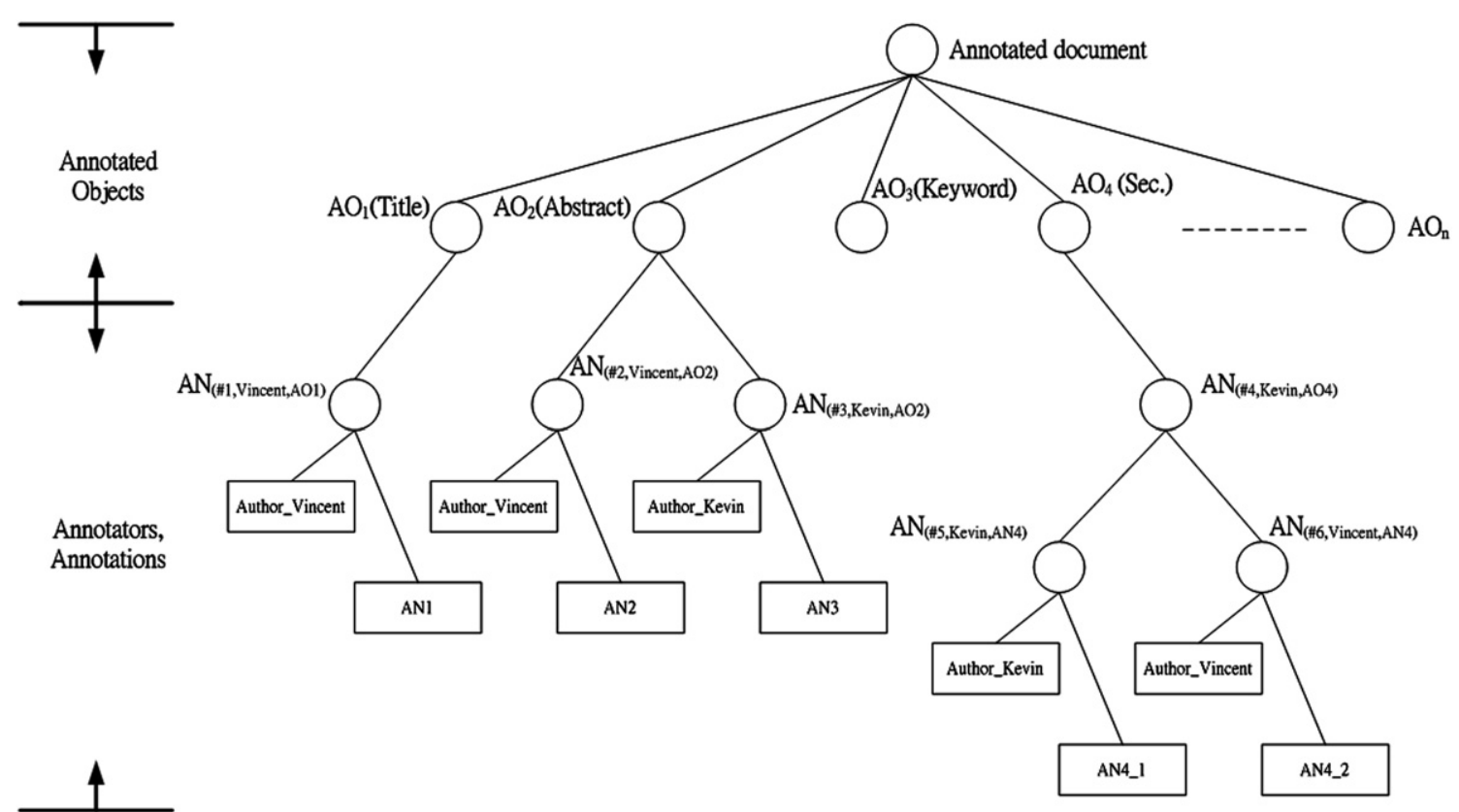

Fig. 2. A tree-like annotation structure.

indicates that Vincent is the only user who made an annotation upon AO1. The other two annotation associations, AN(\#2,Vincent,AO2) and AN(\#3,Kevin,AO2), indicate that Vincent and Kevin made different annotations upon the same annotated object, AO2.

The anchoring position refers to a tree-like annotation structure encapsulating such annotating association within an annotated document. The tree-like annotation structure enables dynamic merging of separated annotations and annotated objects upon user requests, and it is able to provide flexible viewing. The flexible viewing is considered to be one of the key concepts to support collaboration. Users might benefit from the comments and supplements of others as provided through the annotations to an annotated object. Note that annotated objects in a document may overlap with each other.

\subsection{Annotation retrieval}

For personalized (and collaboration), users always have to select a view before content can be loaded and displayed. Users might also remove views, put them to a public directory (to a group of members), or copy a public view for private use and append own annotations to that view. To enhance flexible viewing, this system has developed a simple classification approach (Yang et al., 2004) to semantically organize knowledge. This approach computes the clustering similarity among each annotation with respect to specific concepts, and then classifies the annotations with similar clusters. The clustering concepts here can be denoted by keywords, key phrases, or certain topics based on a standard topic hierarchy. The ACM topic hierarchy (ACM, 1998) acts as a proof-of-concept of our categorizing approach. This mechanism uses the same idea to categorize annotated documents into similar clusters.

According to the annotation management model, this system builds an annotation retrieval handler that dynamically retrieves relevant knowledge based on specific user requirements. A major challenge in this dynamically retrieval is that different users may use variants of a keyword for the same meaning (e.g., plurals, gerund forms, or past tense suffixes), leading to the divergences of different concepts. Porter stemming (Porter, 1980) alleviates this problem by substituting variable names with their respective stems in document parsing. This approach removes common morphological and inflectional endings from English words for term normalization, and produces a similarity value based on the percentage of the number of found keywords against the total number of keywords in the user's query. The procedure of the annotation retrieval is as follows.

Step 1 The annotation retrieval handler waits for incoming user's query with a set of keywords (one to many).

Step 2 Upon receiving a user's query, the association manager computes the semantic similarity between the user's query and each of the clusters in the personalized annotation model, and determines the target cluster with the highest semantic similarity.

Step 3 The association manager confines the user's query and redirects the request to the target cluster in the annotation repository. The semantic similarity between the user's query and each annotation in the target cluster is calculated by comparing normalized keywords. Each result is recorded as a weighted annotation value ( 0.6 for ann_weight) and assigned to the corresponding annotation.

Step 4 The association manager confines the user's query and redirects the request to the target cluster in the annotated document repository. The semantic similarity between the user's query and each annotated document in the target cluster is calculated by comparing normalized keywords. Each result is recorded as a weighted document value ( 0.4 for doc_weight) and assigned to the corresponding document.

Step 5 The association manager checks each existing mapping from the annotation cluster to the document cluster and assigns a weighted similarity value to the "annotate" link, by multiplying the repository weights at the both ends (e.g., annotation $\times$ ann_weight + document $\times$ doc_weight). In this process, if the weighted similarity value outperforms a predefined threshold ( 0.4 for $\mathrm{TH} \_$predefined), then adds the association and document to the final result set. 
Step 6 If the result set is not empty, the annotation retrieval handler returns the user's query results to the user; otherwise, it requests the user to refine the query.

\subsection{Annotation discussion}

To enhance peer interaction, this system designed an annotation discussion handler which is able to help users to exchange their own annotations with others through the proposed annotation model. If a document displays the annotations of all group members, then individuals may ignore them. Further, the privacy of an individual may be invaded by malicious users. Therefore, gathering all annotations to conduct the annotation model provides a good solution to manage individual annotations and group annotations. Group members can review all the annotations in the same group, denoted as $\mathrm{AN}_{u, i}$, where $u=1 \ldots m$, of an annotated object in a document, denoted as $\mathrm{AO}_{i}$. Based on the proposed anchoring position, these annotations can be combined, organized, and displayed clearly. Related discussion can further be treated as a new annotation, denoted as $\mathrm{AN}_{m+1}, i+1$, and associated with an annotated object in a document, i.e. $\mathrm{AO}_{i \text {. }}$

\section{Research objective and methodology}

Although related studies (Hwang et al., 2007; Marshall \& Brush, 2004; Yang et al., 2004) have reported the benefits of collaborative learning with annotation systems, there are still many issues regarding the implementation of Web 2.0 collaborative annotation systems. This study aims at exploring students' annotation behavior and uncovers the relationship between relevant variables (including learning scenarios, learning achievements, and quantity of annotation). Thus, the purpose of this study is to investigate the effects of different annotation sharing scenarios on quantity of annotation and its influence on learning achievements. Furthermore, different annotation sharing scenarios were employed to stimulate students' learning motivation to read, annotate, and share. Finally, this study is to understand students' perceived attitudes toward the use of PAMS 2.0. This study proposes three interesting research questions:

(1) What are students' attitudes toward the use of PAMS 2.0?

(2) What are the effects of different annotation sharing scenarios on quantity of annotation and its influence on learning achievements?

(3) What is the relationship between learning achievements and quantity of annotation?

\subsection{Participants and subjects}

Participants in the experiment class and the control class are 86 freshmen (from National Central University, Taiwan with an average age of 19-20) enrolled in a one-semester first-year computer science course. The experimental course, which was carried out from March 2008 to June 2008, was entitled "Introduction to Computer Science" and took place $3 \mathrm{~h}$ per week in a computer classroom. Furthermore, English was used as first language as a medium of instruction in the two classes. 46 students ( 40 men and 6 women) were assigned to the experiment class, and 40 students ( 36 men and 4 women) were assigned to the control class. They majored in computer science and had basic computer concepts, but had no experience in computer science. Most participants also had experience the use of Web-based discussion board systems.

\subsection{Instruments}

\subsubsection{Self-prepared teaching and learning materials}

This course covers an introductory class for undergraduate computer science majors. The content of this curriculum, which includes software, hardware and networks, resembles that of a one-semester first-year college course. Five topics were arranged in the following sequence. Topic 1 is "Introduction to computer"; Topic 2 is "Basic of computer architecture"; Topic 3 is "Security"; Topic 4 is "Computer networks"; and Topic 5 is "Artificial intelligence".

This course involved face-to-face lectures, and used digital supplemental materials as articles. All participants were asked to study assigned articles and take course tests in different learning scenarios.

\subsubsection{Personalized annotation management system 2.0: PAMS 2.0}

In order to explore the usefulness and effectiveness of PAMS 2.0 in enhancing students' online group reading activity, this system was developed. The Graphical User Interface (GUI) is designed to be simple and clean and focuses on the important functions need to annotate the document. Fig. 3 shows a screenshot of the final GUI. The GUI integrates an input schema editor that allows participators to specify the preferred target format, such as annotation types. This editor supports four annotation types: definitions, comments, questions, and associations. The functionalities provide by the e-document browser panel include highlighting, underlining, textual annotation, as well as voice recording. Students firstly log in to PAMS 2.0 and access the assigned articles. Next, they must identify the format of the target content (e.g., PDF, Word, or Web pages). Students are able to underline, circle, or highlight important passages, or jot down comments in the margin. Also, students use an annotation type as "questions" to represent the meaning of their thoughts. Based on readings of assigned articles, students are required to make unstructured, freeform, idiosyncratic handwriting annotations is critical for sense-making of information. Furthermore, students are required to conduct four annotation types for the content associated with their annotations that they make by reading the article.

In group reading activity, students are required to discuss a common article with group members. By applying this system to inquire, explain, and clarify, they can cooperatively ascertain the meaning of difficult questions. Fig. 4 depicts the annotation discussion handler helps each collaborator's answers and shares them with other collaborators. For example, assume that students were encouraged to raise questions about assigned articles. By using other annotation systems, students must write a lot of words to describe their questions, and then post these words on the website. With PAMS 2.0, they only have to make notes on the article and mark their annotations as "Question" to make them public. As a result, other students can easily obtain the question on the article, and clearly understand the question based on its context. If someone answers the question and marks his/her annotation as a public "Answer," the inquirer can find out the answer by 


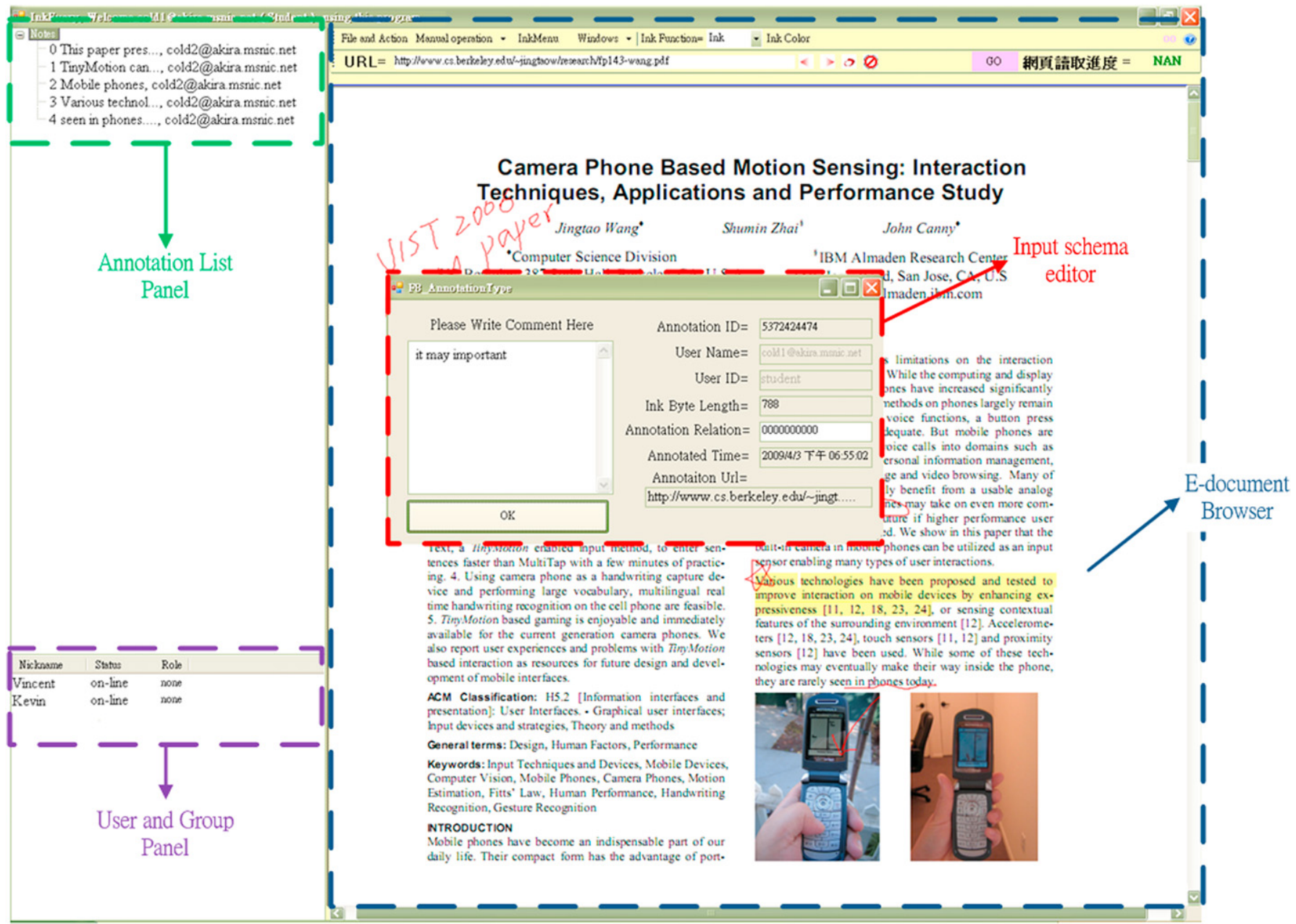

Fig. 3. The Graphical User Interface of PAMS 2.0.

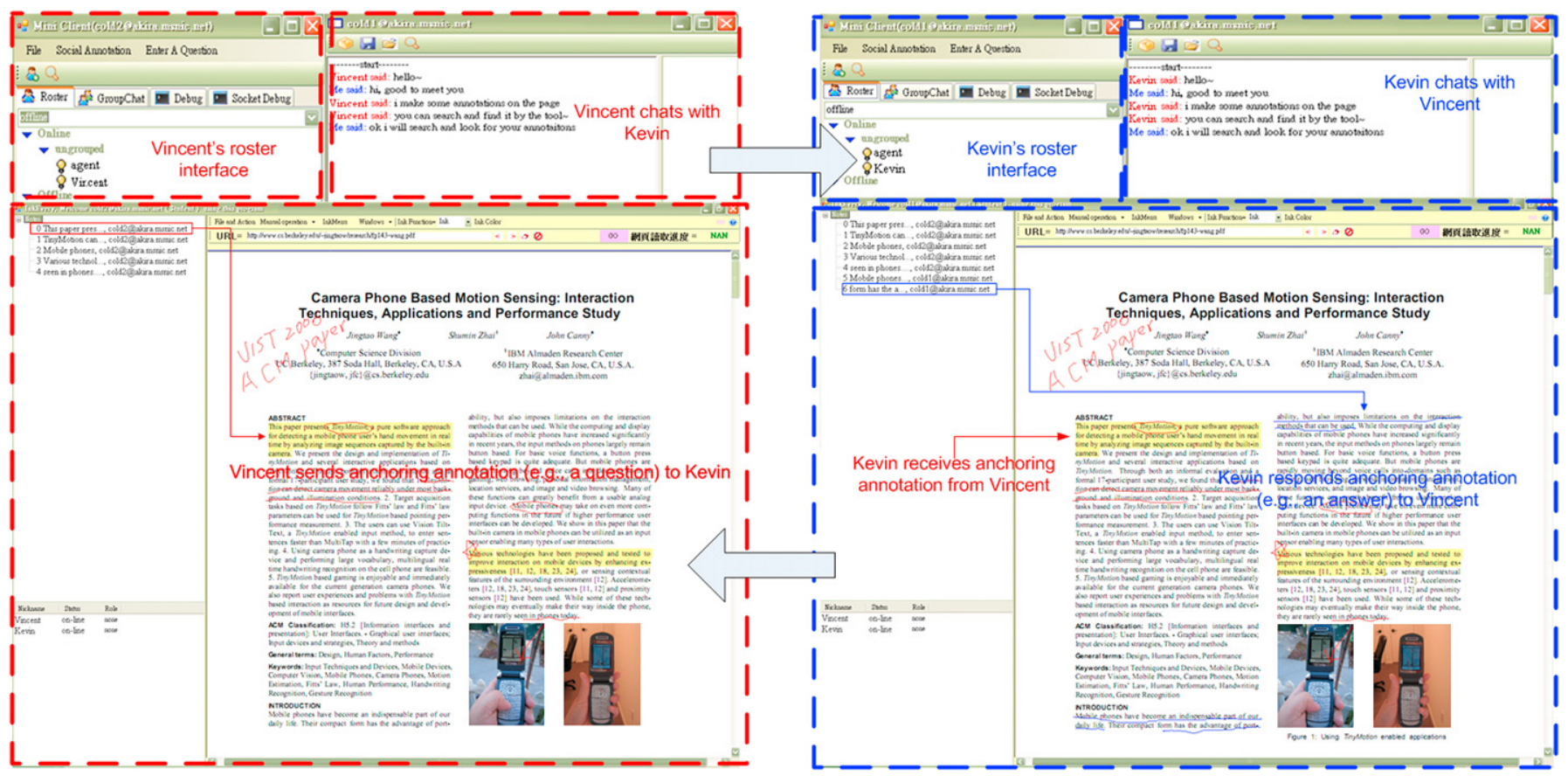

Fig. 4. Students discuss a science article with group members in group reading exercises. 
searching all public annotations on the article using the annotation type of "Answer". With the support of this handler, students can look at each collaborator's answers and choose the best explanation or answer to the problem.

Certain rules can be customized for a specific group discussion. For example, a group may require that each group member must complete their own individual tasks prior to group discussion. If any of group members does not complete his or her tasks, the entire group cannot hold any cooperative discussions. Furthermore, this system can dynamically assign the answering privilege to any group member. Any group member who holds this privilege must submit the group discussion results; if the outcome is not sent by group members, this group cannot to continue its discussion for the next question. Consequently, each group member has an opportunity to submit an answer and be responsible for leading a group discussion.

In addition to learning aids for students, this system also provides a teacher with real-time statistical information about each student learning situation (Casamayor, Amandi, \& Campo, 2009). This system collects information on the learning process of each student and then produces a statistical chart during group reading exercises. Furthermore, in the group discussion, the teacher can focus on a specific student learning status, such as when identifying the article format, giving for each student suggestion and the frequency of looking up learning hints. This statistical information helps the teacher understand each student learning status (for individuals and groups). Additionally, the information can also help the teacher in giving immediate and appropriate feedback to students. Fig. 5(b) shows the statistical data for contributing individual annotations and looking up other collaborators' annotations by a student named "Kevin". Moreover, Fig. 5(c) shows that recording data for a group learning status includes the overall discussion status of each group, the answers to the comprehension questions submitted by each group, and the percentage of scheduled progress achieved. Fig. 5(a) is the interface for assigned learning tasks, and this mechanism would therefore help the teacher to be aware of student learning situation. Also, the teacher can set some options to see the statistical data from this system. This mechanism makes it easy to collect research data for this research.

\subsubsection{Web-based discussion board systems}

In the control class, students used a Web-based discussion board system is MediaWiki (2009), and it is an open and convenient editing system for students in the control class to visit, edit, organize, and update website. Students were encouraged to read assigned articles on the website.

\subsubsection{Other tools}

The researchers also provided several research tools, including pre-tests and post-tests (as described in Section 5.3) and a PAMS 2.0 questionnaire (as described in Section 5.1).

\subsection{Procedures}

The students in two classes were divided into small groups with each group containing two to four students, and each group participated in the arrangement of learning scenarios as shown in Table 1. Five learning scenarios were arranged for five learning topics as well as each topic containing articles and different sharing scenarios. The three and five scenarios were used to explore the influence of the midterm and final exams on learning behavior with the annotation.

Hwang et al. (2007) have emphasized the number of individual annotation. Furthermore, the use of annotation systems should be considered together with group learning activities. The researchers have enhanced the use of PAMS 2.0 with learning scenarios of sharing. Therefore, students in the experiment class were encouraged to make annotations directly on reading articles and to look up other collaborators' annotations. The purpose of this study is to increase the usefulness of PAMS 2.0 through the arrangement of learning scenarios.

The research procedures were described as follows:

(1) Literature review.

(2) Pre-test: A pre-test was kept at the beginning of each scenario to evaluate students' prior knowledge. A comparison of their prior knowledge enabled the learning gains to be assessed.

(3) Experiment class with PAMS 2.0 versus control class without PAMS 2.0: In each learning scenarios lasting three weeks, each group was assigned a group leader, who was in charge of documenting and examining each group member learning situation. In the

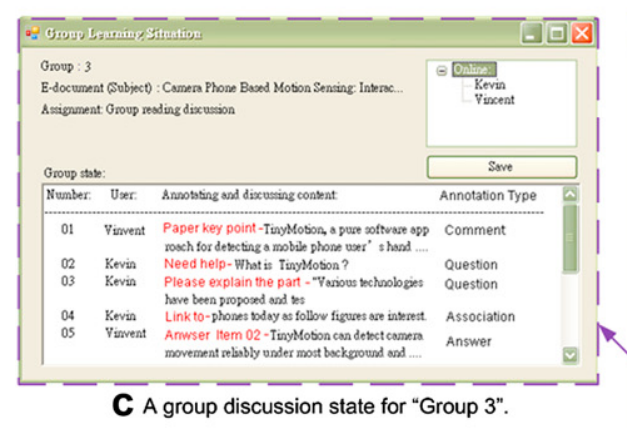

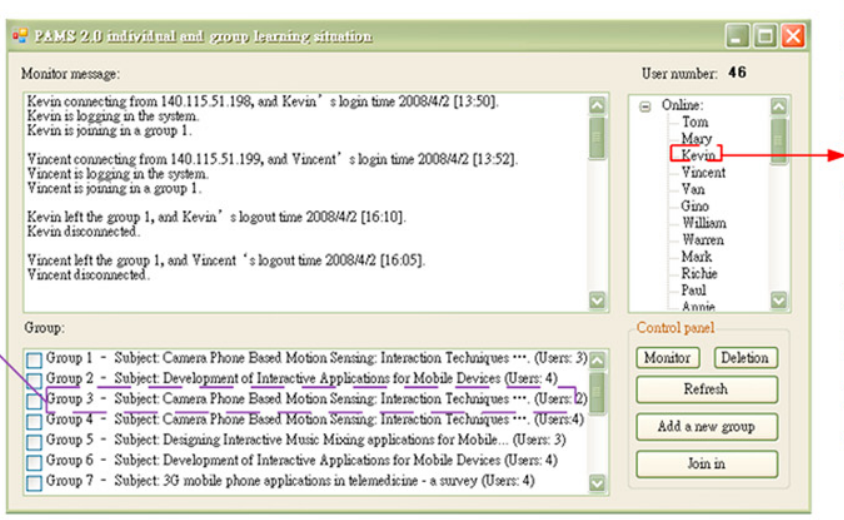

A All users and groups learning situation.

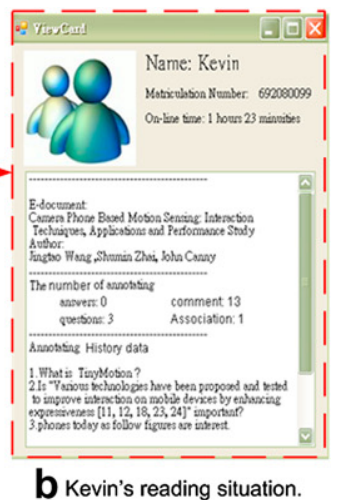

b Kevin's reading situation.

Fig. 5. The interface of student learning situation. 
Table 1

The arrangement of learning scenarios.

\begin{tabular}{|c|c|c|c|}
\hline \# & Experiment class with PAMS 2.0 & \# & Control class without PAMS 2.0 \\
\hline 1. & Group annotation sharing & 1. & Group reading \\
\hline 2. & Group annotation sharing & 2. & Group reading \\
\hline 3. & Group annotation sharing for midterm exam & 3. & Group reading for midterm exam \\
\hline 4. & Group annotation sharing & 4. & Group reading \\
\hline 5. & Group annotation sharing for final exam & 5. & Group reading for final exam \\
\hline
\end{tabular}

experiment class, students could use the PAMS 2.0 to review other collaborators' annotations in a group learning scenario, but they could not view the annotations of the other groups. Students in the control class could use the Web-based discussion board system to view assigned articles in a group learning scenario, but they could not annotate the articles. In contrast, the experiment class was used PAMS 2.0 to clearly explain the meaning of their ideas on annotated articles. Moreover, in order to encourage the students to make more annotations by using PAMS 2.0, and to guide students to view more annotations made by the other collaborators, PAMS 2.0 provides a flexible viewing mechanism which dynamically retrieves relevant annotations based on users' requirements. Moreover, students are industrious to generate more annotations were placed at the annotation list panel. Thus, students could be interested to get a higher position by making more annotations. Additionally, PAMS 2.0 are easy for students to find more useful annotations from the other collaborators to facilitate their reading. However, the control class continued to read and discuss assigned articles collaboratively.

(4) Post-test, midterm exam, and final exam: After the end of the learning scenario, each group leader in two classes turned in a detailed group report, together with a set of questions raised by each group. Accumulated questions from two classes constituted a question bank. We then conducted the post-tests to evaluate learning achievements. After the third and fifth scenarios, the midterm and final exams were concluded to evaluate students' achievements.

(5) PAMS 2.0 questionnaire: After course concluded, a questionnaire was administered to understand students' perceived attitudes toward the use of PAMS 2.0.

(6) Data collection and analysis: This study collected data to propose the research questions. The data include the PAMS 2.0 logs data, pretest and post-test scores, and questionnaire data. In addition, the PAMS 2.0 logs data provided statistical information on the students' annotation behavior while contributing knowledge. For example, the number of annotations (e.g., explanations, arguments, problems, and answers) uploaded by each student, the frequency of looking up learning hints, and the total number of annotations ranked by each student. The statistical information was provided to instructors for monitoring purposes. Moreover, the PAMS 2.0 logs data was employed for investigating the students' knowledge contribution.

\subsection{Research structure and research variables}

This study adopted a quasi-experimental method using learning scenarios as independent variables, and learning achievements as dependent variables. During the experiment, all students learned within the same period, were taught by the same teacher, and utilized the same instructional tool (e.g., the experiment class used PAMS 2.0 and the control class used the Web-based discussion board system) with the same assigned material provided in the course. That is, some variables were controlled.

Fig. 6 illustrates the structure of the research variables. The bidirectional-solid-arrow line shows the correlation between quantity of annotation and learning achievements. The directional-dotted-arrow line means using learning scenarios were investigated to show its influence on learning achievements. Based on the collaborative learning theory, not only PAMS 2.0 was provided for the experiment class to support learning scenarios, but also different annotation sharing scenarios were arranged to increase students' annotation motivations. After the end of each learning scenario, the learning achievements are students' post-test scores. The quantity of each student's annotation was calculated from the PAMS 2.0 logs data.

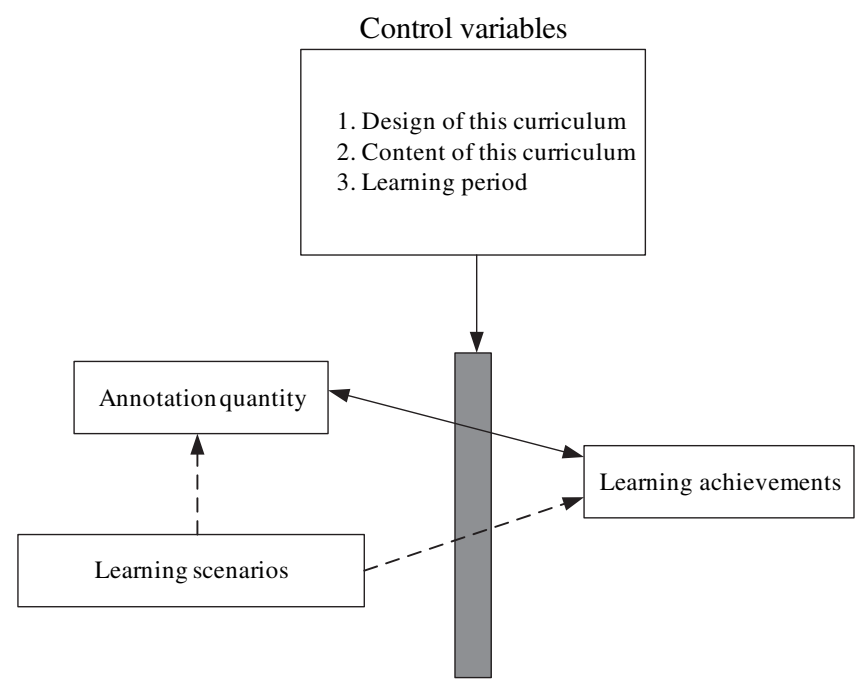

Fig. 6. Research structure and research variables. 


\section{Results and discussions}

\subsection{Reliability and validity of the questionnaire}

The questionnaire was given to 46 students in experiment class and 45 valid answer sheets were returned. This questionnaire was originally developed by Davis (1989) and Azouaou et al. (2004), and modified to evaluate how students come to accept and use PAMS 2.0. This questionnaire included fifteen questions using a five-point Likert scale. The questions can be classified into four dimensions: (1) Perceived usefulness, which examines how PAMS 2.0 helps students learn (five questions); (2) Perceived ease of use, which examines how useful PAMS 2.0 is (five questions); (3) Learning satisfaction, which examines to students' involvement in learning scenarios within PAMS 2.0 (three questions); (4) Willingness for future use, which examines to students' will to use PAMS 2.0 in the future (two questions).

The researchers utilized Cronbach's alpha analysis to evaluate the internal consistency of each dimension of this questionnaire. The Cronbach $\alpha$ values in four dimensions are higher than 0.70 (total Cronbach $\alpha$ value in four dimensions $=0.7983$; the Cronbach $\alpha$ value of "perceived usefulness" $=0.8159$, "perceived ease of use" $=0.7936$, "learning satisfaction" $=0.8019$, and "willingness for future use" $=0.7871$ ). This implies the reliability of this questionnaire is sufficiently high (Wortzel, 1979). During this questionnaire design process, the questions were verified and validated by domain experts. Some ambiguous or unsuitable questions were modified, removed, altered, or arranged in a proper order.

\subsection{Results of the questionnaire analysis}

According to the result of this questionnaire in Table 2, students' attitudes toward the use of PAMS 2.0 can be divided into four different dimensions (perceived usefulness, perceived ease of use, learning satisfaction, and willingness for future use).

In terms of the perceived usefulness, $87 \%$ of the students thought that the annotation types were useful to share their ideas with others in reading groups as indicated in question 1 . In question $2,82 \%$ of the students agreed that the annotation services were useful to express their thoughts freely on online materials. Moreover, regarding question 3, 4, and 5, most of students thought that PAMS 2.0 was useful to exchange their thoughts with others in the same group.

In terms of perceived ease of use, regarding question $6,7,8$, and 9, most of students considered using PAMS 2.0 easy. In question $10,88 \%$ of the students also perceived that it was easy to use PAMS 2.0.

In terms of learning satisfaction, regarding questions 11, 12, and 13, most of students thought that PAMS 2.0 was satisfied with peer interaction. The reasons students preferred learning scenarios were based on whether the activity process did boost their interest, happiness and achievements and whether they helped annotation sharing and learning.

In question $14,89 \%$ of the students would like to continue the use of PAMS 2.0 in the future. The lowest score appeared on question 15 , in which students expressed a negative attitude toward the stability of using PAMS 2.0 in the wireless Internet. Specifically, few students expressed that the connection was unstable. However, some students preferred learning scenarios because they considered PAMS 2.0 easy to use and stable, and especially because PAMS 2.0 did improve interaction between students and annotated articles. In total, most of students thought that PAMS 2.0 was well suited to group learning activities because of its features of lightness, quick turn-on, easy to use, and convenience for collaborative learning environments.

\subsubsection{In comparison with PAMS 2.0 and Web-based discussion board systems}

Most of Web-based discussion board systems use messages to inform the creation of a comment. A well-known example of this collaborative pooling of information is Wikipedia (e.g., MediaWiki), a free online encyclopedia in which the learners contribute the online articles. Furthermore, this mechanism allows the learner to add comments to an online article would allow that resource to grow collaboratively from the accumulated knowledge of the same group members. A more common method for encouraging learners to interact around the content of the online article is the use of Web-based discussion board systems. The use of Web-based discussion board systems can be effective; however, without strong reference to the online article under discussion (Hardaker \& Smith, 2000), the conversation can drift away from relevancy and into argument and trivialities. In contrast, PAMS 2.0 allows collaborative annotations to be made to the content of the articles, and responses to those annotations in Web 2.0 collaborative annotation systems, may provide the benefits of standard annotation while allowing greater collaboration by the increased interaction between learners and online materials (Marshall \& Brush, 2002).

PAMS 2.0 for supporting the individualization of content and Web-based collaborative learning allow bridging the gap between content management and communication. As demonstrated through PAMS 2.0, students might comment on, answer and/or ask questions on the content of online articles, as well as learn in collaborative and highly interactive knowledge-transfer settings without having to care about context-sensitivity and consistency. Additionally, the proposed annotation model provides firsthand feedback from learners to instructors for the purpose of continuous quality management and content improvement. The implemented concept does not only comprise several features for enhancing content for collaborative annotation management, but also the capability to adapt, share and view that are required for individual as well as learning task accomplishment. Incorporating the proposed annotation model with collaborative learning can also be overlaid on multimedia content to enable a new kind of Web 2.0 collaborative annotation system.

\subsection{Analysis of learning achievements}

\subsubsection{Scenario of the first collaborative learning}

5.3.1.1. The results of independent samples T-test: learning achievements. Before this scenario, the teacher prepared a pre-test, which included ten questions, to evaluate students' prior knowledge in the experiment class and the control class. According to the results of the 
Table 2

The PAMS 2.0 questionnaire $(N=45)$.

\begin{tabular}{|c|c|c|c|c|c|c|c|}
\hline \# & Questions & SA & A & $\mathrm{U}$ & $\mathrm{D}$ & SD & Mean \\
\hline \multicolumn{8}{|c|}{ Perceived usefulness } \\
\hline \multirow[t]{2}{*}{1} & I think annotation types (e.g., question, comment, answer, and so on) of & 12 & 27 & 6 & 0 & 0 & 4.13 \\
\hline & PAMS 2.0 were useful to share individual thoughts in a reading group. & $27 \%$ & $60 \%$ & $13 \%$ & $0 \%$ & $0 \%$ & \\
\hline \multirow[t]{2}{*}{2} & I think annotation services (e.g., typing, handwriting, or recording) of & 10 & 27 & 8 & 0 & 0 & 4.04 \\
\hline & PAMS 2.0 were useful to express individual thoughts in online materials. & $22 \%$ & $60 \%$ & $18 \%$ & $0 \%$ & $0 \%$ & \\
\hline \multirow[t]{2}{*}{3} & I think PAMS 2.0 was useful to organize individual or group & 11 & 25 & 9 & 0 & 0 & 4.04 \\
\hline & knowledge in a reading group. & $24 \%$ & $56 \%$ & $20 \%$ & $0 \%$ & $0 \%$ & \\
\hline \multirow[t]{2}{*}{4} & I think PAMS 2.0 was useful to share individual thoughts in a reading group. & 17 & 24 & 3 & 1 & 0 & 4.27 \\
\hline & & $38 \%$ & $53 \%$ & $7 \%$ & $2 \%$ & $0 \%$ & \\
\hline \multirow[t]{2}{*}{5} & I think PAMS 2.0 was useful in a reading group. & 16 & 26 & 3 & 0 & 0 & 4.29 \\
\hline & & $36 \%$ & $58 \%$ & $7 \%$ & $0 \%$ & $0 \%$ & \\
\hline \multicolumn{8}{|c|}{ Perceived ease of use } \\
\hline \multirow[t]{2}{*}{6} & I think it was easy for me to learn how to use PAMS 2.0. & 11 & 29 & 4 & 1 & 0 & 4.11 \\
\hline & & $24 \%$ & $64 \%$ & $9 \%$ & $2 \%$ & $0 \%$ & \\
\hline \multirow[t]{2}{*}{7} & I think the GUI of PAMS 2.0 was easy to use. & 8 & 27 & 9 & 1 & 0 & 3.93 \\
\hline & & $18 \%$ & $60 \%$ & $20 \%$ & $2 \%$ & $0 \%$ & \\
\hline \multirow[t]{2}{*}{8} & It was easy to use PAMS 2.0 to do what I want to do. & 12 & 29 & 4 & 0 & 0 & 4.18 \\
\hline & & $27 \%$ & $64 \%$ & $9 \%$ & $0 \%$ & $0 \%$ & \\
\hline \multirow[t]{2}{*}{9} & I think it was easy for me to use PAMS 2.0 skillfully. & 9 & 28 & 7 & 1 & 0 & 4.00 \\
\hline & & $20 \%$ & $62 \%$ & $16 \%$ & $2 \%$ & $0 \%$ & \\
\hline \multirow[t]{2}{*}{10} & I think the annotation services of PAMS 2.0 were easy to use. & 17 & 27 & 1 & 0 & 0 & 4.36 \\
\hline & & $38 \%$ & $60 \%$ & $2 \%$ & $0 \%$ & $0 \%$ & \\
\hline \multicolumn{8}{|c|}{ Learning satisfaction } \\
\hline \multirow[t]{2}{*}{11} & I think PAMS 2.0 be satisfied with group discussion. & 10 & 30 & 4 & 1 & 0 & 4.09 \\
\hline & & $22 \%$ & $67 \%$ & $9 \%$ & $2 \%$ & $0 \%$ & \\
\hline \multirow[t]{2}{*}{12} & I think PAMS 2.0 be satisfied with group reading activities. & 10 & 30 & 5 & 0 & 0 & 4.11 \\
\hline & & $22 \%$ & $67 \%$ & $11 \%$ & $0 \%$ & $0 \%$ & \\
\hline \multirow[t]{2}{*}{13} & I think PAMS 2.0 be satisfied with group interaction. & 11 & 30 & 4 & 0 & 0 & 4.16 \\
\hline & & $24 \%$ & $67 \%$ & $9 \%$ & $0 \%$ & $0 \%$ & \\
\hline \multicolumn{8}{|c|}{ Willingness for future use } \\
\hline \multirow[t]{2}{*}{14} & I would like to keep using PAMS 2.0 in the future. & 13 & 27 & 3 & 2 & 0 & 4.13 \\
\hline & & $29 \%$ & $60 \%$ & $7 \%$ & $4 \%$ & $0 \%$ & \\
\hline \multirow[t]{2}{*}{15} & I would like to use PAMS 2.0 in other courses. & 7 & 24 & 14 & 0 & 0 & 3.84 \\
\hline & & $16 \%$ & $53 \%$ & $31 \%$ & $0 \%$ & $0 \%$ & \\
\hline Overall mean & & & & & & & 4.11 \\
\hline
\end{tabular}

Note. SA, strongly agree; A, agree; U, undecided; D, disagree; SD, strongly disagree.

independent sample $T$-test, there was no significant difference on the pre-test scores between the experiment class and the control class $(T=-1.155, p=0.251>0.05)$.

After this scenario, the teacher compiled a post-test by selecting thirteen questions from the question bank. As shown in Table 3 , the post-test was given and analyzed with the independent sample $T$-test. The result showed that there was no significant difference between the post-test scores of the two classes. This found that students in the experiment class used PAMS 2.0 in the beginning of the group annotation sharing scenario. Thus, they want to take much time to become familiar with PAMS 2.0 and get benefits in the group annotation sharing scenario.

5.3.1.2. Correlation between learning achievements and quantity of annotation. A Pearson correlation was employed to analyze the correlation between learning achievements and quantity of annotation in the experiment class. There was also no significant positive correlation (the correlation $=0.26, p=0.81>0.05$ ) between learning achievements and quantity of annotation (the quantity of annotation $=463$, mean $=10.07$, $S D=3.997$ ). This is because the experiment class is not familiar with the use of PAMS 2.0 and cannot benefit much from this collaborative facility.

Table 3

The results of independent samples $T$-test: learning achievements.

\begin{tabular}{|c|c|c|c|c|}
\hline Classes & Post-test score & SD & $T$ & $p$ \\
\hline Experiment class & 69.48 & 12.79 & 1.103 & 0.273 \\
\hline Control class & 66.18 & 14.978 & & \\
\hline
\end{tabular}


Table 4

The results of independent samples $T$-test: learning achievements.

\begin{tabular}{|c|c|c|c|c|}
\hline Classes & Post-test score & SD & $T$ & $p$ \\
\hline Experiment class & 72.63 & 8.39 & $2.247^{*}$ & 0.027 \\
\hline Control class & 67.48 & 12.47 & & \\
\hline
\end{tabular}

${ }^{*} p<0.05$.

\subsubsection{Scenario of the second collaborative learning}

5.3.2.1. The results of independent samples T-test: learning achievements. Before this second scenario, a pre-test (ten questions) was given and analyzed with an independent sample $T$-test. The result showed that there was no significant difference between the pre-test scores of the two classes $(T=0.83, p=0.409>0.05)$.

After this second scenario, the post-test (eleven questions) was conducted in two classes. According to the results of the independent sample $T$-test in Table 4, there was significant difference on the post-test scores between the experiment class and the control class. Moreover, the post-test scores of the experiment class were significantly higher than those of the control class. The use of PAMS 2.0 comes to facilitate peer interaction in collaborative learning context, different from the first one because in the second scenario, students in the experiment class know how to view the annotations of the other collaborators and conduct brainstorming to get deeper understanding of online materials and questions with the facility of PAM 2.0.

5.3.2.2. Correlation between learning achievements and quantity of annotation. According to the result of the Pearson correlation, there was a significant positive correlation (the correlation $=0.337, p=0.022<0.05$ ) between learning achievements and quantity of annotation (the quantity of annotation $=608$, mean $=13.22$, $S D=3.04$ ). The correlation of the second scenario is stronger than that of the first scenario. This is because students in the experiment class become familiar with PAMS 2.0, easily create individual annotation, and read the annotations of the other collaborators in collaborative learning environments.

\subsubsection{Scenario of the third collaborative learning for midterm exam}

5.3.3.1. The results of independent samples T-test: learning achievements. In the middle of the semester, a midterm examination was employed to evaluate students' achievements. The midterm exam included fifteen questions and covered three topics taught in this course. According to the results of the independent sample T-test in Table 5, there was no significant difference between the post-test scores of the two classes.

Before this scenario, majority students generally believe that this exam will be the most important factor that determines whether or not they will pass this course, and so they tried their best to study and catch-up materials reading no matter which class they were. Furthermore, students had very high motivation to study for this exam no matter whether instructional tools were provided. Therefore, the midterm exam comes to dominate the students' achievements over instructional tools in the third scenario.

5.3.3.2. Correlation between learning achievements and quantity of annotation. According to the result of the Pearson correlation, there was a significant positive correlation (the correlation $=0.390, p=0.007<0.05$ ) between learning achievements and quantity of annotation (the quantity of annotation $=672$, mean $=14.61, \mathrm{SD}=2.94$ ) in the experiment class. Moreover, the total amount of annotation in this scenario is more than those of the previous two scenarios because the use of PAM 2.0 is stimulated by the midterm exam. This indicated the correlation of the third scenario increased sharply due to most of students had done more catch-up learning tasks for the midterm exam. Thus, PAMS 2.0 can really facilitate learning with collaborative annotations and is worth employing in collaborative learning environments. That is, the more annotations students make using PAMS 2.0, the higher achievement they will obtain.

\subsubsection{Scenario of the fourth collaborative learning}

5.3.4.1. The results of independent samples T-test: learning achievements. Before this fourth scenario, the pre-test (ten questions) was given and analyzed with an independent sample $T$-test. This showed that there was insignificant difference on the pre-test scores between the experiment class and the control class $(T=-1.284, p=0.203>0.05)$.

After the fourth scenario, the post-test (eleven questions) was conducted. According to the result of the independent sample T-test in Table 6, there was significant difference between the post-test scores of the two classes. Moreover, the post-test score of the experiment class was higher than that of the control class. The use of PAMS 2.0 comes to facilitate learning achievements in the group learning scenario.

5.3.4.2. Correlation between learning achievements and quantity of annotation. According to the result of the Pearson correlation, there was a significant positive correlation (the correlation $=0.386, p=0.008<0.05$ ) between students' achievements and quantity of annotation in the experiment class (the quantity of annotation $=583$, mean $=12.67, \mathrm{SD}=3.218$ ). The correlation of the fourth scenario is weaker than that of the third scenario. This result also means the correlation of the fourth scenario was reduced because of the group annotation sharing and learning. Group annotation may reduce students' desire to contribute their annotations, due to easy retrieval of the other collaborator's annotations.

Table 5

The results of independent samples $T$-test: learning achievements (midterm exam).

\begin{tabular}{|c|c|c|c|c|}
\hline Classes & Post-test score & SD & $T$ & $p$ \\
\hline Experiment class & 76.43 & 14.65 & 1.579 & 0.118 \\
\hline Control class & 71.68 & 12.70 & & \\
\hline
\end{tabular}


Table 6

The results of independent samples $T$-test: learning achievements.

\begin{tabular}{|c|c|c|c|c|}
\hline Classes & Post-test score & SD & $T$ & $p$ \\
\hline Experiment class & 77.24 & 9.38 & $2.068^{*}$ & 0.04 \\
\hline Control class & 73.2 & 8.38 & & \\
\hline
\end{tabular}

${ }^{*} p<0.05$.

\subsubsection{Scenario of the fifth collaborative learning for final exam}

5.3.5.1. The results of independent samples T-test: learning achievements. In the end of the semester, the final examination was employed to evaluate learning achievements. A total of twenty-five questions were prepared for the final exam, including the last two topics taught in this course. According to the result of the independent sample T-test in Table 7, there was no significant difference between the post-test scores of the two classes.

The post-test scores in final scenario were much higher than those in other scenarios, respectively. This is because in the final exam, students had very high motivation to study for passing this course. Like the third scenario for midterm exam, no matter whether instructional tools were provided, students would study hard for the final exam. This exam comes to dominate students' achievements over assisted any instructional tools in this scenario. This indicated that appropriate learning activity design should be considered to facilitate the usefulness of PAM 2.0 to students' achievements. In the future study, some peer assessment for annotation may be employed to help students get more and deeper understanding of online material.

5.3.5.2. Correlation between learning achievements and quantity of annotation. According to the result of the Pearson correlation, there was a significant positive correlation (the correlation $=0.416, p=0.004<0.05$ ) between learning achievements and quantity of annotation in the experiment class (the quantity of annotation $=738$, mean $=16.04, \mathrm{SD}=3.483$ ). The quantity of annotation in the fifth scenario is higher than that in other scenarios. It is clear that student had done more catch-up learning tasks for the final exam.

\subsection{Summary of results}

This questionnaire revealed that PAMS 2.0 played a critical role in enhancing learning with collaborative annotations effects and helping students share their own annotations among one another. From the items of usefulness, most of students thought that annotation systems do improve their online reading performance. For example, students can communicate rapidly using Ink annotations in any language, as well as handwritings or drawings, without having to learn the typing method of the keyboard-based computer. From the items of ease of use, most of students also perceived that it is easy to use annotation systems. From the items of learning satisfaction, most of the students thought that group learning scenarios with annotation systems did increase their interest, happiness and achievements in collaborative learning context; also they thought annotation systems did improve interaction between learners and annotated documents. According to the result of this questionnaire in Table 2, students in the experiment class have positive attitudes toward the use of PAMS 2.0 and are willing to use PAMS 2.0 in the future. This result corresponds to the requirements of PAMS 2.0 for collaborative learning context (Azouaou et al., 2004).

This study designed several learning scenarios to evaluate learning achievements in the experiment class and the control class. Regarding the result of the first scenario in Table 3, there was no significant difference between learning achievements of the experiment class and the control class. This is because the experiment class is not familiar with the use of PAMS 2.0 and cannot benefit much from this collaborative facility. Regarding the results of the second scenario in Table 4 and the fourth scenario in Table 6 , there was significant difference between the learning achievements of the experiment class and the control class. This implied that the use of PAMS 2.0 in the two scenarios appears to raise more questions, give meaningful answers, repeat discussions, and elaborate solutions. Moreover, the use of PAMS 2.0 comes to help students' achievements in collaborative learning context. Thus, the results indicated that learners who are willing to do real learning task with PAMS 2.0, and are able to elaborate what they are doing, produce both highest quality annotations and learning achievements. Regarding the results of the third scenario in Table 5 and the fifth scenario in Table 7 , there was no significant difference between the learning achievements of the experiment class and the control class. The lack of a significant difference for the midterm exam and the final exam is interesting. Does the control class have to do more catch-up learning tasks? As we know, students generally believe that the midterm exam and the final exam will be the most important factor that determines whether they will pass this course, and so they would study hard for the two exams. Thus, it is possible that the experiment class and the control class were highly motivated by the two exams, even though instructional tools were provided. This factor appeared to dominate learning achievements over instructional tools in the third scenario and the fifth scenario. Moreover, the quantity of annotation in the fifth scenario is higher than that in other scenarios. This implied that students had done more catch-up learning tasks for the midterm exam and the final exam.

This study finding is similar to that obtained by Hwang et al. (2007). Researchers reported that the most of students felt that the use of annotation systems enhanced their learning tasks and the test results for those students with access to annotation systems were significantly increased compared to the control class. However, the result of the final exam was similar between the experiment class and the

Table 7

The results of independent samples $T$-test: learning achievements (final exam).

\begin{tabular}{|c|c|c|c|c|}
\hline Classes & Post-test score & SD & $T$ & $p$ \\
\hline Experiment class & 82.11 & 12.17 & 1.199 & 0.233 \\
\hline Control class & 79.45 & 7.11 & & \\
\hline
\end{tabular}


control class to suggest that there was a "ceiling effect due to high motivation to study for the final exam", although it is noted that it may be that the control class had to study more intensely to obtain this result in the final exam that the group with access to annotation systems. The amount of increase in a student's achievement in the post-test showed a direct correlation with the level of sharing activity with the students who annotated more frequently achieving the higher marks; however, students with higher motivation are the most likely to make frequent annotations and this may account for the observed difference.

\section{Conclusions}

This study developed a Personalized Annotation Management System 2.0 (PAMS 2.0), as a Web 2.0 collaborative annotation system, for managing, sharing, and reusing individual/collaborative annotations as well as providing a shared mechanism for discussion about shared annotations among multiple users. From the experiment results of the practical computer science course, several interesting and important findings were derived.

Firstly, the latest Web 2.0 technology has enabled universities and corporations to reach out and educate students across time and space barriers. Although this technology promotes Web-based collaborative learning activities, understanding students' perceptions toward the use of PAMS 2.0 is still a critical issue. According to the results of this questionnaire, most of students have positive attitudes toward the questions for four dimensions (perceived usefulness, perceived ease of use, learning satisfaction, and willingness for future use). Students would like to use PAMS 2.0 in group learning scenarios because of they believe PAMS 2.0 easy to use and stable. Thus, most of students thought that PAMS 2.0 helped them think carefully about understanding collaborators' annotations with annotated documents in collaborative learning context.

Secondly, this study designed several learning scenarios to evaluate learning achievements in the experiment class and the control class. The analytical results of learning achievements show that the use of the PAMS 2.0 system is able to increase students' achievements in the second and fourth scenarios, and this finding is similar to that obtained by Hwang et al. (2007). Researchers consider that students have the willingness to utilize collaborative annotations in online articles reading have higher achievements in collaborative learning environments. In addition, regarding the results of two scenarios, it indicates a positive correlation between learning achievements and quantity of annotation. This is because students in the experiment class are familiar with the use of PAMS 2.0 so that they can raise more questions, give meaningful answers, repeat discussions, and elaborate solutions. Furthermore, students make more annotation also have higher achievements in collaborative learning environments.

Finally, according to the results of the third scenario and the fifth scenario, there is no significant difference between learning achievements of the experiment class and the control class. This implied that in two particular scenarios (for the midterm and final exams), the high learning motivation of students to study for the two exams produced results for the control class that are similar to the experiment class. In addition, regarding the results of two particular scenarios, it also indicates a positive correlation between learning achievements and quantity of annotation. The total number of annotations in two particular scenarios is larger than the number of previous other scenarios' annotation, it is because the use of PAMS 2.0 has been stimulated by midterm and final exams. This implies the correlation of two particular scenarios increased mainly because most of the students had done more catch-up learning tasks in midterm and final examinations. In addition, there is a ceiling effect due to high learning motivation to study for midterm and final exams no matter what kind of instructional tools or learning scenarios were provided. This phenomenon is still to be explored. Although some possible factors (including the design of this curriculum, learning period, teacher, assigned articles, and more) that could influence the use of instructional tools and learning scenarios have been discussed, no major factors or reasons for this phenomenon can be found in this research. This is worth exploring through further research.

\section{Acknowledgements}

This work is supported by National Sceince Council, Taiwan under grants NSC96-2628-S-008-008-MY3, NSC98-2511-S-008-006-MY3 and NSC98-2511-S-008-007-MY3.

\section{References}

ACM. ACM computing classification system toc. Retrieved 1998. Available from. http://www.aifb.uni-karlsruhe.de/WBS/pha/bib/acmtopics.rdf.

Annotea. Retrieved 2001. Available from. http://www.w3.org/2001/Annotea/.

Avouris, N., Komis, V., Margaritis, M., \& Fiotakis, G. (2004). An environment for studying collaborative learning activities. Educational Technology and Society, 7(2), 34-41.

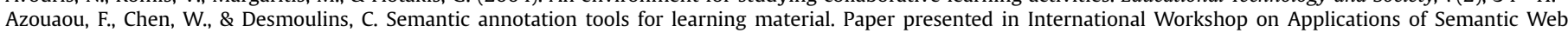
Technologies for E-Learning, 2004.

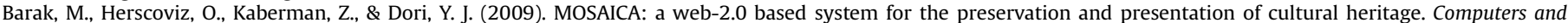
Education, 53(3), 841-852.

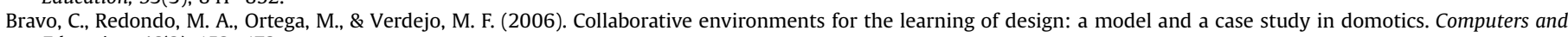
Education, 46(2), 152-173.

Casamayor, A., Amandi, A., \& Campo, M. (2009). Intelligent assistance for teachers in collaborative e-learning environments. Computers and Education, 53(4), 1147-1154.

Davis, F. D. (1989). Perceived usefulness, perceived ease of use, and user acceptance of information technology. MIS Quarterly, 13(1), 319-340.

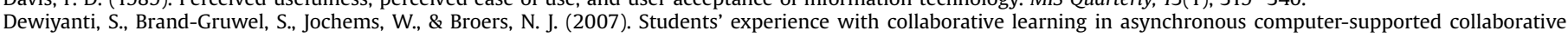
learning environments. Computers in Human Behavior, 23(1), 496-514.

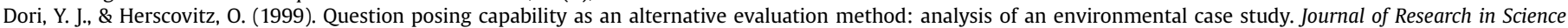
Teaching, 36(4), 411-430.

Dublin Core. Dublin core metadata initiative. Retrieved 2001. Available from. http://dublincore.org.

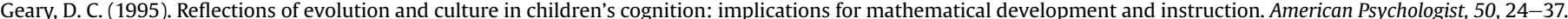

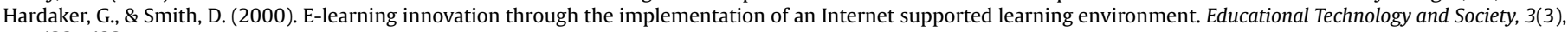
$422-432$.

Hwang, W. Y., Wang, C. Y., \& Sharples, M. (2007). A study of multimedia annotation of web-based materials. Computers and Education, 48(4), 680-699.

Jones, N., Blackey, H., Fitzgibbon, K., \& Chew, E. (2010). Get out of MySpace! Computers and Education, 54(3), 776-782.

Kagan, S. (1994). Cooperative learning. San Clemente, California: Kagan Publishing.

Koschmann, T. (1996). Paradigm shifts and instructional technology: an introduction. CSCL: Theory and Practice of an Emerging Paradigm. 
LeeTiernan, S., \& Grudin, J. (2001). Fostering engagement in asynchronous learning through collaborative multimedia annotation. Proceedings of Interact 472-479.

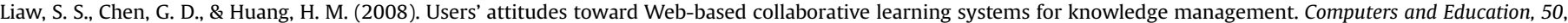
950-961.

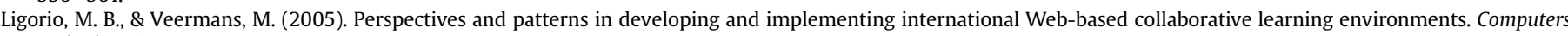
and Education, 45, 271-275.

LOM. Learning object metadata. Retrieved 2002, from. http://ltsc.ieee.org/wg12/index.html.

Marshall, C. C. (1997). Annotation: From paper books to the digital library. Proceedings of the second ACM conference on digital libraries, $131-140$.

Marshall, C. C. (1998). Toward an ecology of hypertext annotation. Proceedings of the ninth ACM conference on Hypertext and hypermedia, 40-49.

Marshall, C. C., \& Brush, A. J. (2002). Exploring the relationship between personal and public annotations. ACM Press, 349-357.

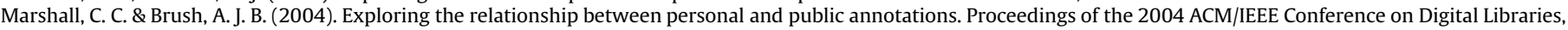
349-357.

MediaWiki. Retrieved 2009, from. http://www.mediawiki.org/wiki/MediaWiki.

Nardi, B. A. (1996). Context and consciousness. Activity theory and human-computer interaction. MIT Press.

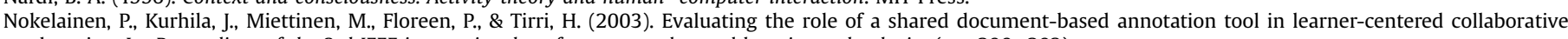
learning. In. Proceedings of the 3rd IEEE international conference on advanced learning technologies (pp. 200-203).

Porter, M. (1980). An algorithm for suffix stripping program. Automated Library and Information Systems, 14(3), 130-137.

Robert, C. A. (2009). Annotation for knowledge sharing in a collaborative environment. Journal of Knowledge Management, 13(1), 111-119.

Sandhu, R., Coyne, E. J., Feinstein, H. L., \& Youman, C. E. (1996). Role-based access control models. IEEE Computer, $29(2), 38-47$.

Schunk, D. H. (1996). Learning theories. New Jersey: Prentice-Hall.

SCORM. Advanced distributed learning network shareable content object reference model. Retrieved 2003, from. http://www.adlnet.org/.

Vygotsky, L. (1978). Mind in society: the development of higher psychological processes. Cambridge, MA: Harvard University Press.

Wang, Q. (2009). Design and evaluation of a collaborative learning environment. Computers and Education, 53(4), 1138-1146.

Wortzel, R. (1979). New life style determinants of women's food shopping behavior. Journal of Marketing, 43, 28-29.

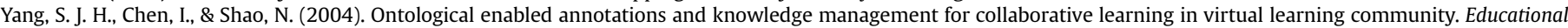
Technology and Society, 7(4), 70-81.

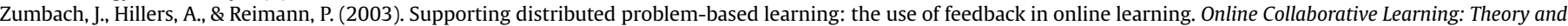
Practice 86-103.

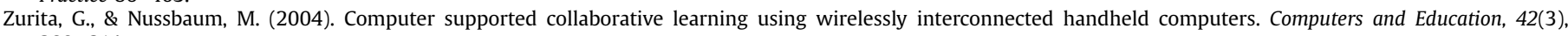
$289-314$. 